\title{
Relationships between Neighborhood Walkability and Objectively Measured Physical Activity Levels in Hemodialysis Patients
}

\author{
Maggie Han ${ }^{a} \quad$ Xiaoling Ye $^{a} \quad$ Priscila Preciado $^{a} \quad$ Schantel Williams $^{\mathrm{a}}$ \\ Israel Campos ${ }^{a}$ Marcee Bonner $^{b}$ Candace Young ${ }^{b}$ Daniel Marsh ${ }^{b}$ \\ John W. Larkin ${ }^{c, d}$ Len A. Usvyat ${ }^{d}$ Franklin W. Maddux ${ }^{d}$ \\ Roberto Pecoits-Filho ${ }^{c}$ Peter Kotanko ${ }^{a, e}$ \\ a Renal Research Institute, New York, NY, USA; ${ }^{b}$ Renal Associates, Baton Rouge, LA, USA; ${ }^{c}$ Pontificia Universidade \\ Catolica do Parana, Curitiba, Brazil; ${ }^{\mathrm{d}}$ Fresenius Medical Care North America, Waltham, MA, USA; ${ }^{\mathrm{e}}$ cahn School of \\ Medicine, New York, NY, USA
}

\section{Keywords}

End-stage renal disease - Chronic kidney disease .

Hemodialysis - Walkability · Physical activity · Activities of daily living

\begin{abstract}
Background/Aims: Neighborhood walkability is associated with indicators of health in the general population. We explored the association between neighborhood walkability and daily steps in hemodialysis (HD) patients. Methods: We measured daily steps over 5 weeks using Fitbit Flex (Fitbit, San Francisco, CA, USA) and retrieved Walk Score ${ }^{\circledR}$ (WS) data by patient's home ZIP code (www.walkscore.com; $0=$ poorest walkability; 100 = greatest walkability). Results: $\mathrm{HD}$ patients took a mean of 6,393 $\pm 3,550$ steps/day $(n=46)$. Median WS of the neighborhood where they resided was 28. Patients in an above-median WS $(n=27)$ neighborhood took significantly more daily steps compared to those $(n=19)$ in a below-median WS neighborhood (7,514 $\pm 3,900$ vs. 4,800 \pm 2,228 steps/day; $p<0.001, t$ test). Daily steps and WS were directly correlated $(R=0.425 ; p=0.0032$, parametric test; $R=$ $0.359, p=0.0143$, non-parametric test). Conclusion: This is the first study conducted among HD patients to indicate a
\end{abstract}

(c) 2018 S. Karger AG, Basel

\section{E-Mail karger@karger.com www.karger.com/bpu \\ KARGER}

direct relationship between neighborhood walkability and the actual steps taken. These results should be considered when designing initiatives to increase and improvise exercise routines in HD populations.

(c) 2018 S. Karger AG, Base

\section{Background}

Sedentary lifestyles are associated with increased risk for onset/exacerbation of morbidities and all-cause mortality in the general population, especially for cardiovascular diseases [1]. Living a physically active life is recommended to reduce these risks $[1,2]$. Hemodialysis (HD) patients have an increased cardiovascular mortality and it has been shown that they are less active than their healthy counterparts [2-7]. Some common morbidities in HD populations have been linked to physical inactivity, such as anemia, bone mineral disorders, cardiovascular abnormalities, inflammation, malnutrition, and depression $[4-6,8,9]$. Urbanicity of residence (i.e., urban, suburban, rural) as well as neighborhoods environment have been shown to be associated with physical activity levels in the general and HD populations [10-14]. Despite 
this, specific neighborhood characteristics have in some cases not been identified to be associated with physical activity levels in older adults and those with chronic illness [15-17].

Walkability indexes are measures of neighborhood characteristics that are conducive of higher levels of physical activity; these metrics have been found to be surrogate markers of physical activity levels and associated with health outcomes in the general population [10-13, $18,19]$. The Walk Score ${ }^{\circledR}$ (WS; www.walkscore.com) is a publicly available index of neighborhood characteristics that are conducive to walking in the United States, Cana$\mathrm{da}$, and Australia. The WS measures neighborhood characteristics on a scale of 0 (poorest walkability) to 100 (greatest walkability) based on distance and a variety of key public and commercial destinations (e.g., grocery stores, retail stores, parks, schools). The WS has been validated to be correlated with objective neighborhood walkability indexes based on geographic information systems data [20], and has been demonstrated to be related to physical activity levels and associated with health outcomes in the general population $[19,21,22]$. It has been shown in the general population that the probability of people meeting physical activity recommendations increases by $26 \%$ for every 10 -point increase in the WS [19]. Currently, it is unknown whether neighborhood characteristics are associated with physical activity levels in patients who are affected by end-stage renal disease (ESRD). Understanding the factors that influence physical activity levels in people with ESRD is of great importance to designing optimal support systems and targeted interventions to increase physical health and improve health outcomes in this population.

Objectives

We hypothesized that the walkability of a neighborhood is related to, and a marker of physical activity levels in people with ESRD undergoing HD. Hence, the aims of this investigation were to assess whether neighborhood walkability determined using the WS is associated to and correlated with objectively measured physical activity levels in HD patients residing in urban and suburban environments in the United States.

\section{Methods}

\section{Study Design}

This retrospective study included the analysis of physical activity data previously collected from 2 clinical research studies conducted on maintenance HD patients from both an urban (New York, NY, USA) and suburban (Baton Rouge, LA, USA) area, us- ing the Fitbit ${ }^{\circledR}$ Flex ${ }^{\mathrm{TM}}$ (Fitbit, San Francisco, CA, USA) device for a duration of 5 weeks $[14,23]$. We included patients who previously participated in one of 2 clinical trials conducted at Fresenius kidney care clinics located in the New York City borough of Manhattan, NY (NYC) from September 2014 to May 2015, or clinics located in Baton Rouge, LA (BR) from February 2016 to August 2016. The protocols were approved by Western Institutional Review Board (protocol numbers: 20171534 [NYC] and 20152105 $[\mathrm{BR}])$.

\section{Participants}

Participants who consented to participate met the following inclusion criteria for the original trials: receiving HD treatment thrice weekly for more than 3 months, age $\geq 18$ and $\leq 75$ years old, able to walk without assistive devices, and English speaking. Patients were excluded from the original trials if they had an acute infection, congestive heart failure (CHF; NYHA class 4) and/or unstable angina, were hospitalized $<3$ months prior to enrollment for non-access related reasons, were known to exhibit allergies to nickel, and/or had a cognitive impairment. Demographic data (e.g., age, race, and gender) were captured from the participants' electronic health records (EHRs).

\section{Physical Activity Data}

To capture physical activity levels in the initial trials, HD patients were equipped with a wrist-based accelerometer activity tracker (Fitbit ${ }^{\circledR}$ Flex $^{\mathrm{TM}}$ ) for 5 weeks. During the observational period, participants were instructed to wear the activity tracker at all times, except during their routine HD treatments. The activity trackers were charged and the activity data were downloaded during the participants' HD treatments. At the end of the 5-week observational period, activity data (steps taken and distance traveled) were aggregated and exported from the Fitbit website using their Premium software feature.

\section{Walkability Data}

The WS utilizes public data available from sources such as Google, Education.com, Open Street Map, and Localeze to calculate the distance and variety of key public and commercial destinations, and assigns a score based off the reference location (e.g., geographic coordinates, address, zip code). The key destinations are classified in the following categories: dining and drinking establishments, grocery stores, retail shopping stores, businesses for routine errands, parks and gyms, schools, entertainment, and culture. The WS uses an algorithm to compute the linear combination of weighted distances to destinations in each category, and the results are normalized to fit a scale from 0 (poorest walkability) to 100 (greatest walkability). For each category, amenities within a quarter-mile are assigned a score of 100 , and amenities greater than one mile away are assigned a score of zero. For this study, we captured data on the participants WS in their zip code of residence in January of 2017.

\section{Clinical and Laboratory Data}

Standard of care laboratory data (Spectra Laboratories, USA) was captured from the EHR for the analysis. Additionally, inflammatory serum markers, pre-albumin, and C-reactive protein (CRP), were measured in these studies by Spectra Clinical Research, USA from pre-dialysis blood samples collected on the midweek dialysis session. 
Table 1. Cohort descriptive statistics

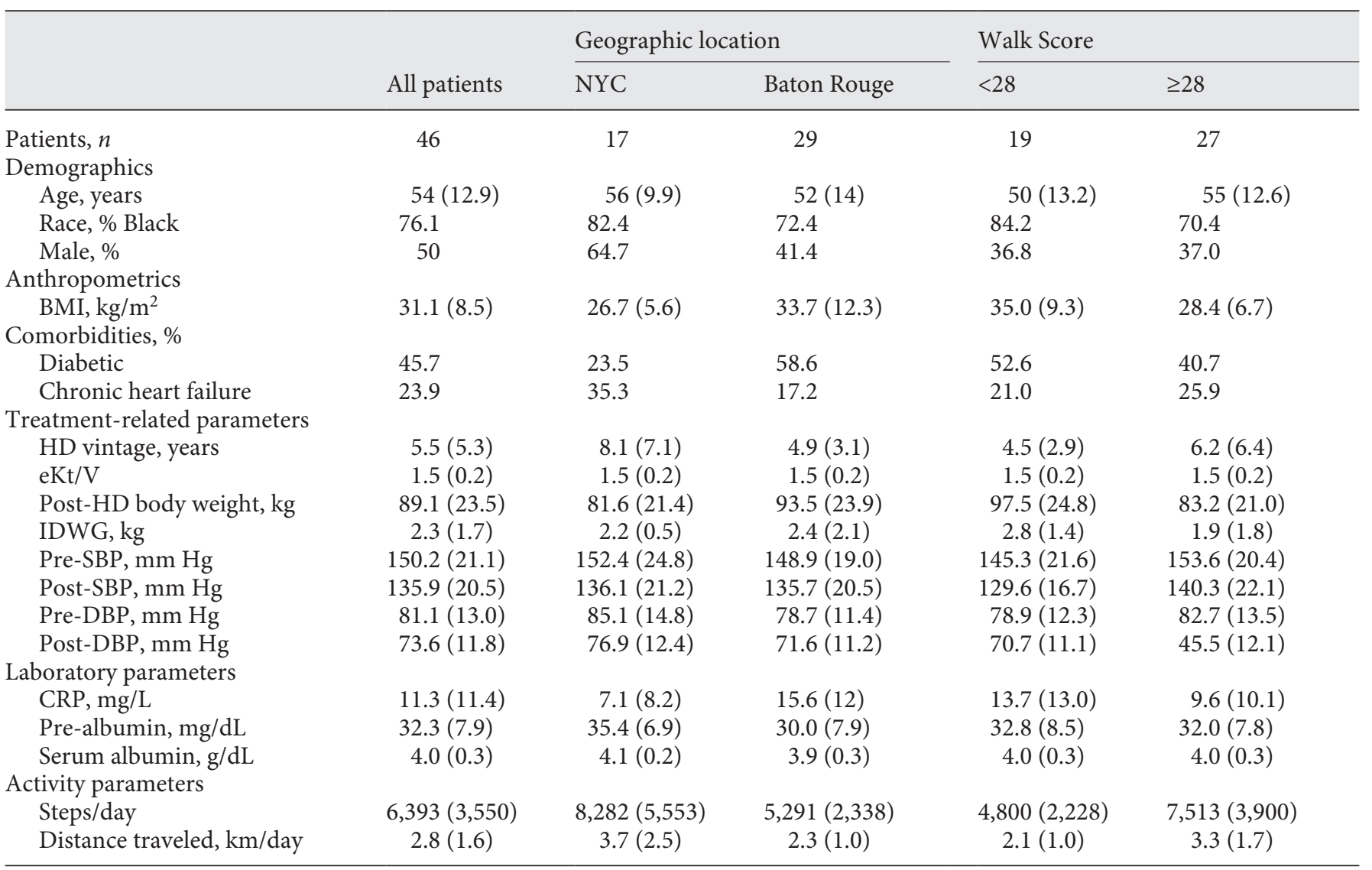

NYC, New York City; BMI, body mass index; HD, hemodialysis; IDWG, interdialytic weight gain; SBP, systolic blood pressure; DBP, diastolic blood pressure; CRP, C-reactive protein.

Patient level data on covariates including HD vintage, total equilibrated HD dose (eKdrt/V), post-HD weight, interdialytic weight gain, pre-/post-HD systolic and diastolic blood pressure were captured from EHRs.

\section{Comorbidities}

$\mathrm{CHF}$ and diabetes mellitus were defined using ICD-9 and/or ICD-10 codes documented in the patients' EHR.

\section{Analysis Design}

Descriptive statistics comprised of means $( \pm S D)$ for continuous variables and percentages for categorical variables. The mean, $\mathrm{SD}$, and median WS was calculated for the entire population and the NYC and BR cohorts. Parametric and non-parametric linear correlation and regression analyses were performed to examine the association between daily average steps and WS using the zip code of each patient's residence for the total population, as well as for the NYC and BR cohort. Patients were then stratified into 2 groups depending on them being above (high walkability) or below (low walkability) the median WS. In order to discern the association between WS and physical activity patterns, average steps per day on HD days (days of HD treatment), non-HD days (days without HD treatment except Sundays), and Sundays were computed for the low and high walkability groups, as well as the entire population. Paired $t$-test methods were used to compare differences in the step count for HD days, non-HD days, and Sundays. Differences between the means and 95\% CI were estimated for step counts on HD days, non-HD days, and Sundays.

\section{Results}

\section{Patient Characteristics}

In total, data from $46 \mathrm{HD}$ patients were analyzed. Of those, 17 were from NYC and 29 from BR. Patient characteristics are depicted in Table 1 . The mean age was 54.0 \pm 12.9 years, dialysis vintage was $5.5 \pm 5.3$ years, $50 \%$ were male, $76.1 \%$ were black, $45.7 \%$ had diabetes, and $23.9 \%$ had CHF. Patients took an average of 6,393 $\pm 3,550$ steps/ 


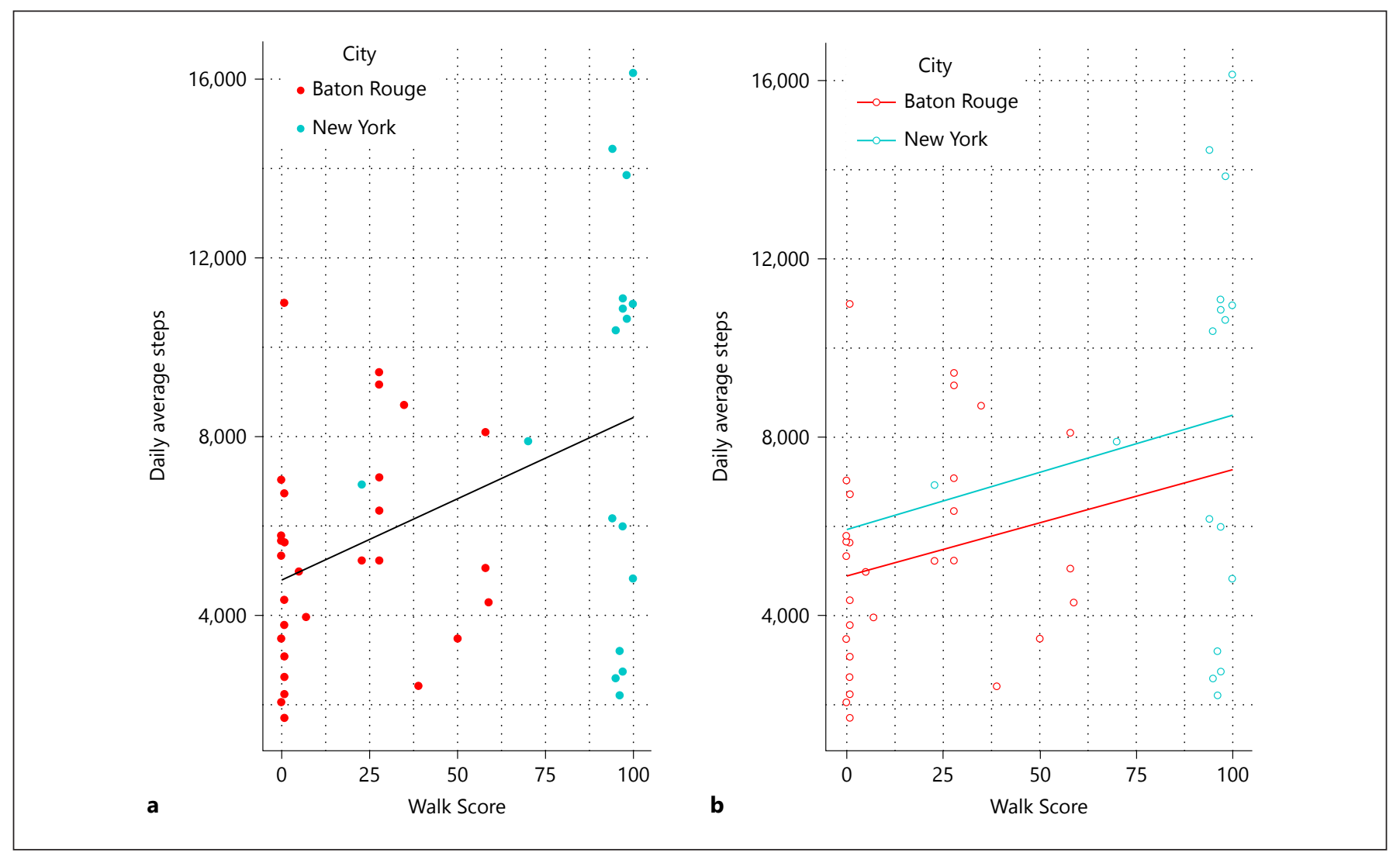

Fig. 1. Walk Score (WS) vs. average steps per day. a Combined BR and NYC data sets. Line of best fit: steps/day $=37^{*}$ WS $+4,781$. b Separated BR and NYC data sets NYC line of best fit: steps/day $=26^{*}$ WS $+5,924$. BR line of best fit: steps/day $=24 *$ WS $+4,895$.

day; those residing in NYC took more steps/day than patients in BR $(8,282 \pm 5,553$ steps/day vs. 5,291 $\pm 2,338$ steps/day, $\Delta 2,991$ steps/day [95\% CI 218-5,764]).

\section{Association of Physical Activity and WS}

The mean WS for all subjects, NYC subjects, and BR subjects was $44 \pm 41,91 \pm 19$, and $17 \pm 20$ respectively. Both parametric $(\mathrm{R}=0.425 ; p=0.0032)$ and nonparametric analysis $(\mathrm{R}=0.359, p=0.0143)$ indicated a positive correlation between the WS and average daily steps (Fig. 1). The direct linear relationship is preserved when the 2 cohorts, NYC and BR, are analyzed separately with NYC's line of best fit exhibiting an upward shift, which reflects the higher level of physical activity in that group.

When stratified into 2 groups by median WS of 28,19 subjects were in the low walkability group (WS $<28$ ) and 27 subjects in the high walkability group (WS $\geq 28$; Table 1). Patients in the low walkability group took significantly less steps per day than those in the high walkability group $(4,800 \pm 2,228$ vs. $7,514 \pm 3,900$, respectively, $p<0.001)$.
Assessment of activity patterns identified that patients in the low walkability group took significantly less steps per day on HD days and non-HD days compared to the high walkability group (Fig. 2). There were no differences between groups for steps per day on Sundays. There were also no differences between steps per day during the days of the week within the low and high walkability groups.

We expanded our analysis by exploring physical activity patterns related to the WS neighborhood description (walkscore.com). To that end, we conducted a separate analysis by "walkable" (WS $\geq 50$ ) or "car-dependent" (WS $<50$ ) neighborhoods, with 26 patients in car-dependent and 20 patients in walkable neighborhoods. As in the case of using the median WS cut-off of 28, patients in the WS $<50$ group walked significantly less on HD days than those in the WS $\geq 50$ group. However, there were no difference in the steps taken on non-HD days between the 2 groups. Those with a WS $<50$ took significantly less steps per day on HD days compared to Sundays (Fig. 3). 


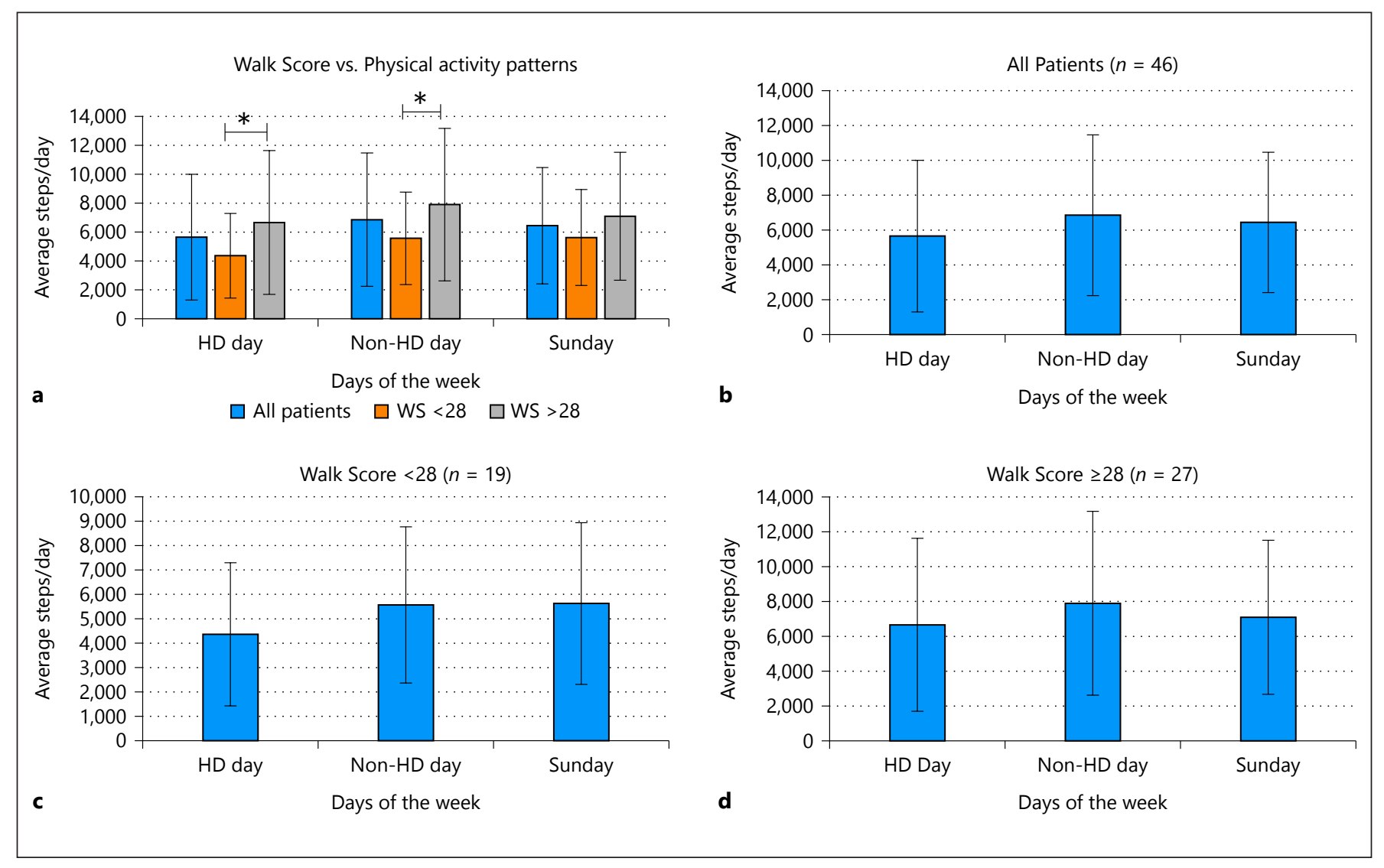

Fig. 2. Association of physical activity patterns and Walk Score (WS; ${ }^{*} p<0.05$ ). a WS vs. physical activity patterns across 3 groups: all patients, those with WS less than median (WS <28), and those with WS greater than median (WS $\geq 28$ ). b Average steps/day of

Patient Characteristics Relative to Walkability Groups Using the cut-off of WS $<28$, we compared clinical, laboratory, and treatment variables between patients in the low and high walkability groups (Table 1). Patients in the high walkability group were older $(55.0 \pm 12.6$ years high walkability vs. $50.0 \pm 13.2$ years low walkability), had longer HD vintage $(6.2 \pm 6.4$ high walkability vs. $4.5 \pm 2.9$ years low walkability), lower body mass index (BMI; $28.4 \pm 6.7 \mathrm{~kg} / \mathrm{m}^{2}$ high walkability vs. $35.0 \pm 9.3 \mathrm{~kg} / \mathrm{m}^{2} \mathrm{low}$ walkability), higher prevalence of CHF (25.9\% high walkability vs. $21.0 \%$ low walkability), lower interdialytic weight gain $(1.9 \pm 1.8 \mathrm{~kg}$ high walkability vs. $2.8 \pm 1.4 \mathrm{~kg}$ low walkability), and higher pre and post blood pressures. When considering the inflammation status, patients in the high walkability group exhibited lower CRP levels $(9.6 \pm 10.1 \mathrm{mg} / \mathrm{L}$ high walkability vs. $13.7 \pm 13.0 \mathrm{mg} / \mathrm{L} \mathrm{low}$ walkability), yet had similar levels of pre-albumin and albumin levels. all patients on HD day, non-HD day, and Sunday. c Average steps/ day of WS $<28$ on HD day, non-HD day, and Sunday. d Average steps/day of WS $\geq 28$ on HD day, non-HD day, and Sunday.

\section{Discussion}

The findings of this study identify for the first time that the walkability of a neighborhood is positively correlated to and associated with physical activity levels in HD patients. These observations are consistent with those of previous studies in the general population $[19,21]$; however, there is a contrast in some select findings in elderly people and those with chronic illnesses including hearing and vision impairments, and musculoskeletal diseases [15-17]. Our study showed that patients who live in more walkable areas exhibited significantly higher physical activity levels compared to those who live in less walkable areas, even though they were older, had a higher prevalence of $\mathrm{CHF}$, and longer HD vintage. To date, the relationships between neighborhood walkability and physical activity have not been explored among HD populations. 


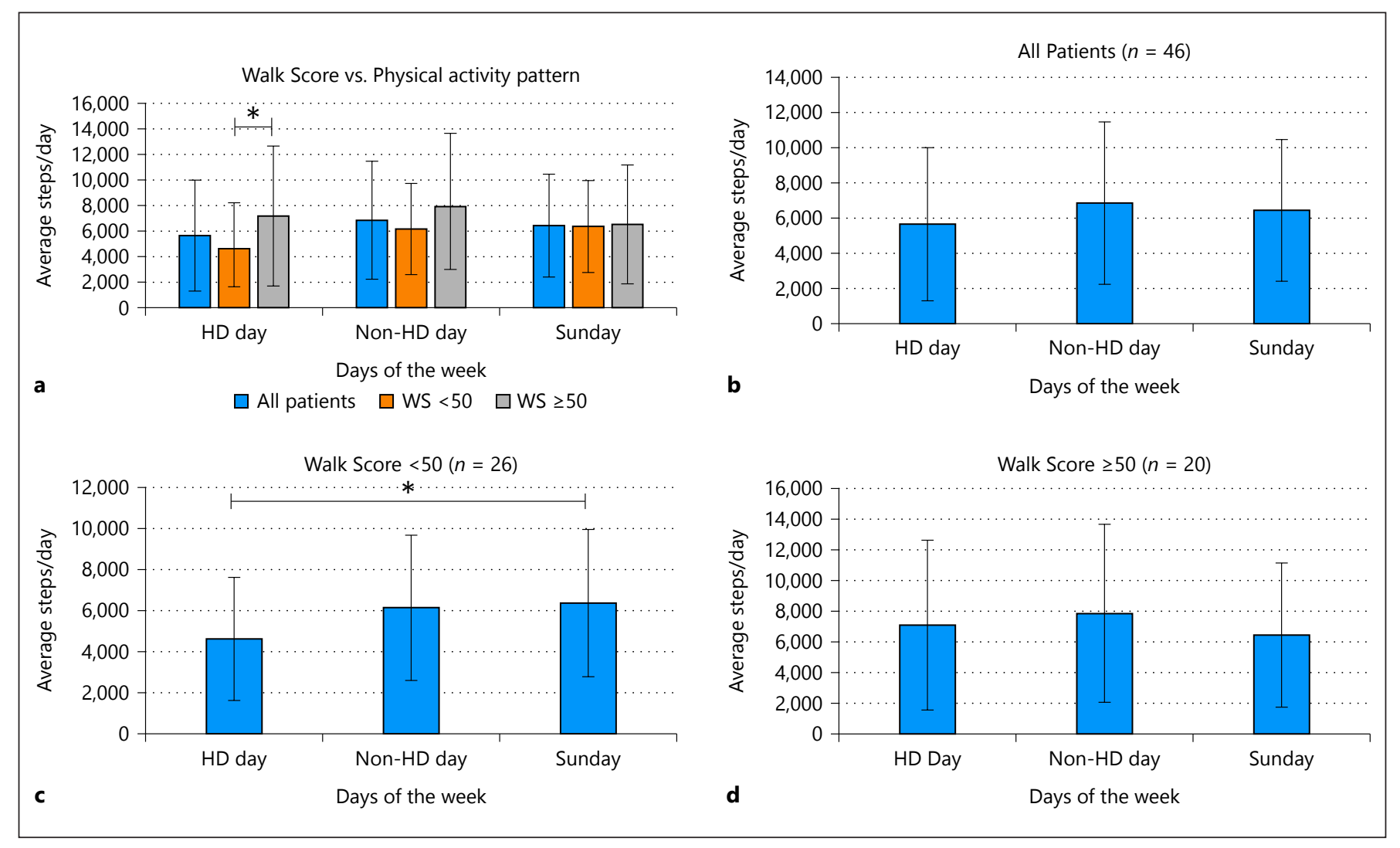

Fig. 3. Association of physical activity patterns and Walk Score (WS; ${ }^{*} p<0.05$ ). a WS vs. physical activity patterns across 3 groups: all patients, those with WS $<50$, and those with WS $\geq 50$. b Average steps/day of all patients on HD day, non-HD day, and Sunday.

HD patients commonly have sedentary lifestyles that increase their risk for an array of negative outcomes, and compounds upon their existing risk for cardiovascular morbidity and mortality $[4,6,7,14,24-26]$. O'Hare et al. [27] studied a large cohort of 4,024 incident $\mathrm{HD}$ and peritoneal dialysis patients and observed that patients with sedentary lifestyles exhibited a $62 \%$ higher risk of oneyear mortality (HR 1.62; 95\% CI 1.16-2.27) compared with patients with active lifestyles. Neighborhood environment could play an important role in physical activity levels. The ease of performing routine daily activities by walking or riding a bike to amenities such as grocery stores has the potential to impact non-leisure physical activity patterns. Neighborhood environment appears to be a crucial factor for dialysis providers and clinicians to consider when optimizing and prioritizing interventions to improve physical activity levels in HD patients.

Physical inactivity in HD patients may be secondary to the burden of ESRD and its associated negative impacts, c Average steps/day of WS $<50$ on HD day, non-HD day, and Sunday. d Average steps/day of WS $\geq 50$ on HD day, non-HD day, and Sunday. including inflammatory responses and nutritional deficiency. The release of cytokines can stimulate a catabolic state and cause irreversible muscle breakdown. In a study of self-reported physical activity levels in dialysis patients, the doubling of physical activity levels was associated with an increase of $3 \mathrm{mg} / \mathrm{dL}$ in pre-albumin levels and a decrease of $40-50 \%$ in CRP levels [28]. Our findings are somewhat consistent with this possible explanation. Patients in the low walkability group (WS <28) showed more signs of inflammation (mean CRP $=13.7 \mathrm{mg} / \mathrm{L}$ ) compared to the high walkability group (WS $\geq 28$; mean $\mathrm{CRP}=9.6 \mathrm{mg} / \mathrm{L}$ ). Despite this, pre-albumin and albumin levels did not differ by high and low neighborhood walkability.

Notably, the HD treatment itself promotes catabolism and patients are commonly known to have post-dialysis fatigue, which could impact physical activity levels. It has been observed in HD patients that physical activity levels are typically lower on HD days, as compared to non-HD 


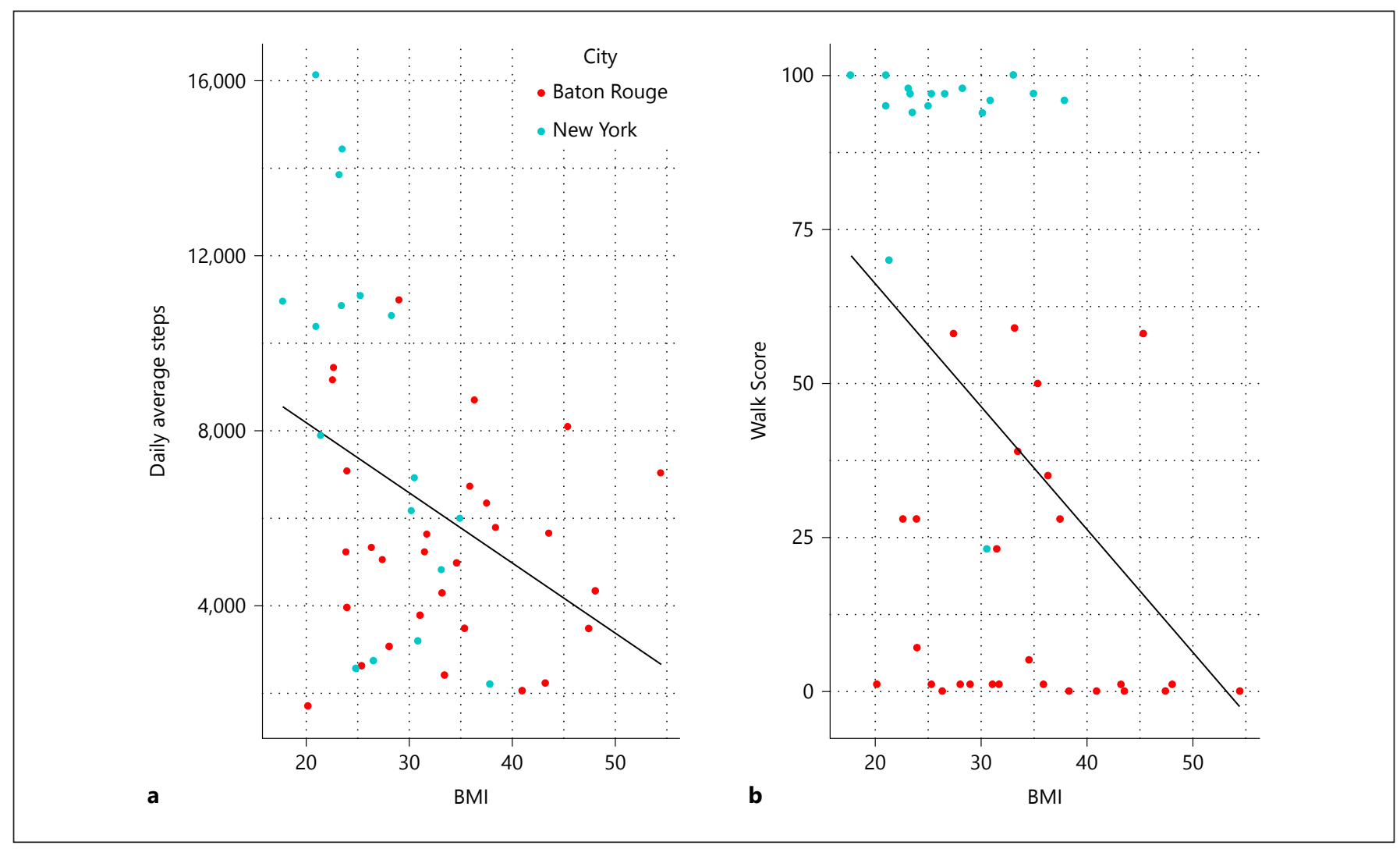

Fig. 4. Association of BMI $\left(\mathrm{kg} / \mathrm{m}^{2}\right)$ with daily average steps and Walk Score (WS). a BMI vs. daily average steps; line of best fit: daily average steps $=-1.99 * \mathrm{BMI}+106$. $\mathbf{b}$ BMI vs. WS; line of best fit: WS $=-160 * \mathrm{BMI}+11,377$.

days $[14,29]$. In our study, we found that patients residing in more walkable areas took significantly more steps per day on HD days, as compared to those residing in less walkable areas. This characteristic is present independent of whether a WS $\geq 28$ or WS $\geq 50$ was considered "more" walkable. Patients in the high walkability group, independent to WS cut off, performed a similar number of steps per day on HD days, non-HD days, and Sundays, and this supports our hypothesis that living in more walkable areas has a positive effect on physical activity levels. Patients residing in a neighborhood with a WS $<50$ took significantly more steps per day on Sundays compared to HD days, yet there were no differences in steps per day observed between those residing in a neighborhood with a WS $<28$.

Higher levels of physical activity have been established to be related to better weight management in ESRD patients [4-6]. In an exploratory sub-analysis in this study, we identified correlations between higher mean steps per day and lower BMI ( $r=0.1667 ; p=0.0048)$, as well as between higher WS and BMI ( $r=0.1457 ; p=0.0089$; Fig. 4$)$. Our observations provide insight for the development of programs to increase physical activity levels in HD patients. Ultimately, our results suggest that HD patients residing in areas with poor walkability may gain the most benefit from paradigms to improve physical activity levels on dialysis days, such as use of intradialytic exercise programs. Alternatively, in patients who reside in areas that are more conducive to walking, different physical activity improvement paradigms may be the most optimal, such as initiatives that aim to increase exercise throughout the week.

\section{Limitations}

Although this study has many strengths and identified novel findings, it does have several limitations that include a small population of patients that are not representative of the overall HD population. Additionally, the study population largely included patients in areas with a very low or very high WS (NY and BR) and did not have an even distribution of geographies.

Another limitation of this study is that it used combined data from 2 clinical research studies that objective- 
ly tracked activity levels in patients randomized to either a control group, or an intervention that included the provision of routine reports on the patients' activity levels $[14,23]$. Therefore, the influences of interventions may have affected activity levels in interventional versus control patients at each study site location. However, the intervention was not identified to alter the number of steps performed in the original studies and any effects would be anticipated to be balanced for each specific geographical population. Further research is needed to confirm the findings of this study.

\section{Conclusions}

The findings indicate that neighborhood walkability is related to and a marker of physical activity levels and patterns in HD patients. Higher neighborhood WS is associated with higher levels of physical activity regardless of the HD treatment day patterns. Albeit further studies are warranted to confirm these observations, these findings should be considered by dialysis providers and clinicians when designing interventions to increase and improvise the exercise routine in the ESRD population.

\section{Disclosure Statement}

This research was funded by Renal Research Institute. M.H., X.Y., S.W., I.C., P.P., J.W.L., L.A.U., F.W.M., and P.K. are employees of Fresenius Medical Care North America, or the subsidiary company Renal Research Institute. M.B., C.Y., and D.M. are employees of Renal Associates. R.P.-F. is an employee of and J.W.L. is a student at Pontificia Universidade Catolica do Parana. P.K. is an employee of Renal Research Institute. L.A.U., P.K., and F.W.M. have stock ownership in Fresenius Medical Care. P.K. receives honoraria from UpToDate, and is on the Editorial Board of Blood Purification and Kidney and Blood Pressure Research. R.P.-F. receives research grants, consulting fees, and honoraria from Baxter Healthcare and Fresenius AG. F.W.M. has directorships in American National Bank and Trust (NASDAQ: AMNB), Mid-Atlantic Renal Coalition, Specialty Care, Inc., and Sound Physicians, is the Chairman of Pacific Care Renal Foundation 501c3 nonprofit, is the Chair of Kidney Care Partners, and the founder of Scholarships Expanding Education 501c3 nonprofit.

\section{References}

1 Physical Activity Guidelines Advisory Committee: Physical Activity Guidelines Advisory Committee Report. Washington, US Department of Health and Human Services, 2008.

2 Tudor-Locke C, Craig CL, Aoyagi Y, Bell RC, Croteau KA, De Bourdeaudhuij I, Ewald B, Gardner AW, Hatano Y, Lutes LD, Matsudo SM, Ramirez-Marrero FA, Rogers LQ, Rowe DA, Schmidt MD, Tully MA, Blair SN: How many steps/day are enough? For older adults and special populations. Int J Behav Nutr Phys Act 2011;8:80.

3 Saran R, Robinson B, Abbott KC, Agodoa LY, Albertus P, Ayanian J, Balkrishnan R, BraggGresham J, Cao J, Chen JL, Cope E, Dharmarajan S, Dietrich X, Eckard A, Eggers PW, Gaber C, Gillen D, Gipson D, Gu H, Hailpern SM, Hall YN, Han Y, He K, Hebert H, Helmuth M, Herman W, Heung M, Hutton D, Jacobsen SJ, Ji N, Jin Y, Kalantar-Zadeh K, Kapke A, Katz R, Kovesdy CP, Kurtz V, Lavalee D, Li Y, Lu Y, McCullough K, Molnar MZ, MontezRath M, Morgenstern H, Mu Q, Mukhopadhyay $\mathrm{P}$, Nallamothu B, Nguyen DV, Norris KC, O'Hare AM, Obi Y, Pearson J, Pisoni R, Plattner B, Port FK, Potukuchi P, Rao P, Ratkowiak K, Ravel V, Ray D, Rhee CM, Schaubel DE, Selewski DT, Shaw S, Shi J, Shieu M, Sim JJ, Song P, Soohoo M, Steffick D, Streja E, Tamura MK, Tentori F, Tilea A, Tong L, Turf M, Wang D, Wang M, Woodside K, Wyncott A, Xin X, Zang W, Zepel L, Zhang S, Zho H, Hirth RA, Shahinian V: US renal data system
2016 annual data report: epidemiology of kidney disease in the United States. Am J Kidney Dis 2017;69(3 suppl 1):A7-A8

4 Lopes AA, Lantz B, Morgenstern H, Wang M, Bieber BA, Gillespie BW, Li Y, Painter P, Jacobson SH, Rayner HC, Mapes DL, Vanholder RC, Hasegawa T, Robinson BM, Pisoni RL: Associations of self-reported physical activity types and levels with quality of life, depression symptoms, and mortality in hemodialysis patients: the DOPPS. Clin J Am Soc Nephrol 2014;9:1702-1712.

5 Morishita S, Tsubaki A, Shirai N: Physical function was related to mortality in patients with chronic kidney disease and dialysis. Hemodial Int 2017;21:483-489.

6 Johansen KL, Chertow GM, Ng AV, Mulligan K, Carey S, Schoenfeld PY, Kent-Braun JA: Physical activity levels in patients on hemodialysis and healthy sedentary controls. Kidney Int 2000;57:2564-2570.

7 Panaye M, Kolko-Labadens A, Lasseur C, Paillasseur JL, Guillodo MP, Levannier M, Teta D, Fouque D: Phenotypes influencing low physical activity in maintenance dialysis. J Ren Nutr 2015;25:31-39.

8 Afsar B: The relationship between intact parathyroid hormone levels and daily physical activity in hemodialysis patients. Int J Artif Organs 2014;37:350-357.

9 Zamojska S, Szklarek M, Niewodniczy M, Nowicki M: Correlates of habitual physical activity in chronic haemodialysis patients. Nephrol Dial Transplant 2006;21:1323-1327.
10 Sallis JF, Cerin E, Conway TL, Adams MA, Frank LD, Pratt M, Salvo D, Schipperijn J, Smith G, Cain KL, Davey R, Kerr J, Lai PC, Mitas J, Reis R, Sarmiento OL, Schofield G, Troelsen J, Van Dyck D, De Bourdeaudhuij I, Owen N: Physical activity in relation to urban environments in 14 cities worldwide: a crosssectional study. Lancet 2016;387:2207-2217.

11 Christian H, Knuiman M, Divitini M, Foster S Hooper P, Boruff B, Bull F, Giles-Corti B: A Longitudinal analysis of the influence of the neighborhood environment on recreational walking within the neighborhood: results from RESIDE. Environ Health Perspect 2017;125:077009.

12 Siqueira Reis R, Hino AA, Ricardo Rech C, Kerr J, Curi Hallal P: Walkability and physical activity: findings from Curitiba, Brazil. Am J Prev Med 2013;45:269-275.

13 Althoff T, Sosic R, Hicks JL, King AC, Delp SL, Leskovec J: Large-scale physical activity data reveal worldwide activity inequality. Nature 2017;547:336-339.

14 Williams S, Han M, Ye X, Zhang H, MeyringWosten A, Bonner M, Young C, Thijssen S, Marsh D, Kotanko P: Physical activity and sleep patterns in hemodialysis patients in a suburban environment. Blood Purif 2017;43:235-243.

15 King AC, Salvo D, Banda JA, Ahn DK, Chapman JE, Gill TM, Fielding RA, Demons J, Tudor-Locke C, Rosso A, Pahor M, Frank LD: Preserving older adults' routine outdoor activities in contrasting neighborhood environments through a physical activity intervention. Prev Med 2017;96:87-93. 
16 King AC, Sallis JF, Frank LD, Saelens BE, Cain K, Conway TL, Chapman JE, Ahn DK, Kerr J: Aging in neighborhoods differing in walkability and income: associations with physical activity and obesity in older adults. Soc Sci Med 2011;73:1525-1533.

17 Barnett A, Cerin E, Zhang CJP, Sit CHP, Johnston JM, Cheung MMC, Lee RSY: Associations between the neighbourhood environment characteristics and physical activity in older adults with specific types of chronic conditions: the ALECS cross-sectional study. Int J Behav Nutr Phys Act 2016;13:53.

18 Creatore MI, Glazier RH, Moineddin R, Fazli GS, Johns A, Gozdyra P, Matheson FI, Kaufman-Shriqui V, Rosella LC, Manuel DG, Booth GL: Association of neighborhood walkability with change in overweight, obesity, and diabetes. JAMA 2016;315:2211-2220.

19 Brown SC, Pantin H, Lombard J, Toro M, Huang S, Plater-Zyberk E, Perrino T, PerezGomez G, Barrera-Allen L, Szapocznik J: Walk score ${ }^{\circledR}$ : associations with purposive walking in recent Cuban immigrants. Am J Prev Med 2013;45:202-206.

20 Duncan DT, Aldstadt J, Whalen J, Melly SJ, Gortmaker SL: Validation of walk score for estimating neighborhood walkability: an analysis of four US metropolitan areas. Int J Environ Res Public Health 2011;8:4160-4179.

21 Towne SD Jr, Lopez ML, Li Y, Smith ML, Warren JL, Evans AE, Ory MG: Examining the role of income inequality and neighborhood walkability on obesity and physical activity among low-income hispanic adults. J Immigr Minor Health 2017, Epub ahead of print.

22 Mazumdar S, Learnihan V, Cochrane T, Phung H, O'Connor B, Davey R: Is walk score associated with hospital admissions from chronic diseases? Evidence from a cross-sectional study in a high socioeconomic status Australian city-state. BMJ Open 2016;6: e012548.
23 Han M, Williams S, Mendoza M, Ye X, Zhang H, Calice-Silva V, Thijssen S, Kotanko P, Meyring-Wosten A: Quantifying physical activity levels and sleep in hemodialysis patients using a commercially available activity tracker. Blood Purif 2016;41:194-204.

24 Kutner NG, Zhang R, Allman RM, Bowling CB: Correlates of ADL difficulty in a large hemodialysis cohort. Hemodial Int 2014;18:7077.

25 Tudor-Locke C, Washington TL, Hart TL: Expected values for steps/day in special populations. Prev Med 2009;49:3-11.

26 Locatelli F, Marcelli D, Conte F, D'Amico M, Del Vecchio L, Limido A, Malberti F, Spotti D: Cardiovascular disease in chronic renal failure: the challenge continues. Registro Lombardo Dialisi e Trapianto. Nephrol Dial Transplant 2000;15(suppl 5):69-80.

27 O'Hare AM, Tawney K, Bacchetti P, Johansen KL: Decreased survival among sedentary patients undergoing dialysis: results from the dialysis morbidity and mortality study wave 2 . Am J Kidney Dis 2003;41:447-454.

28 Anand S, Chertow GM, Johansen KL, Grimes B, Kurella Tamura M, Dalrymple LS, Kaysen GA: Association of self-reported physical activity with laboratory markers of nutrition and inflammation: the Comprehensive Dialysis Study. J Ren Nutr 2011;21:429-437.

29 Majchrzak KM, Pupim LB, Chen K, Martin CJ, Gaffney S, Greene JH, Ikizler TA: Physical activity patterns in chronic hemodialysis patients: comparison of dialysis and nondialysis days. J Ren Nutr 2005;15:217-224.

30 Katayama A, Miyatake N, Nishi H, Ujike K, Hashimoto H, Kurato R, Koumoto K: Relationship between changes in physical activity and changes in health-related quality of life in patients on chronic hemodialysis with 1-year follow-up. Acta Med Okayama 2016;70:353361.

31 Gomes EP, Reboredo MM, Carvalho EV, Teixeira DR, Carvalho LF, Filho GF, de
Oliveira JC, Sanders-Pinheiro H, Chebli JM, de Paula RB, Pinheiro Bdo V: Physical activity in hemodialysis patients measured by triaxial accelerometer. Biomed Res Int 2015; 2015:645645.

32 Carvalho EV, Reboredo MM, Gomes EP, Teixeira DR, Roberti NC, Mendes JO, Oliveira JC, Sanders-Pinheiro $\mathrm{H}$, Pinheiro BV: Physical activity in daily life assessed by an accelerometer in kidney transplant recipients and hemodialysis patients. Transplant Proc 2014;46:1713-1717.

33 Shibata S, Tsutou A, Shiotani H: Relation between sleep quality and daily physical activity in hemodialysis outpatients. Kobe J Med Sci 2014;59:E161-E166.

34 Matsuzawa R, Matsunaga A, Kutsuna T, Ishii A, Abe Y, Yoneki K, Harada M, Ishibashi M, Takeuchi Y, Yoshida A, Takahira N: Association of habitual physical activity measured by an accelerometer with high-density lipoprotein cholesterol levels in maintenance hemodialysis patients. ScientificWorldJournal 2013;2013:780783.

35 Avesani CM, Trolonge S, Deleaval P, Baria F, Mafra D, Faxen-Irving G, Chauveau P, Teta D, Kamimura MA, Cuppari L, Chan M, Heimburger O, Fouque D: Physical activity and energy expenditure in haemodialysis patients: an international survey. Nephrol Dial Transplant 2012;27:2430-2434

36 Shimoda T, Matsuzawa R, Yoneki K, Harada M, Watanabe T, Matsumoto M, Yoshida A, Takeuchi Y, Matsunaga A: Changes in physical activity and risk of all-cause mortality in patients on maintence hemodialysis: a retrospective cohort study. BMC Nephrol 2017;18: 154.

37 Cupisti A, Capitanini A, Betti G, D’Alessandro C, Barsotti G: Assessment of habitual physical activity and energy expenditure in dialysis patients and relationships to nutritional parameters. Clin Nephrol 2011;75:218-225. 